\title{
Bistable lasing in twin microdisk photonic molecules
}

\author{
Satoru Ishii and Toshihiko Baba ${ }^{\text {a) }}$ \\ Department of Electrical and Computer Engineering, Yokohama National University, 79-5 Tokiwadai, \\ Hodogayaku, Yokohama 240-8501, Japan
}

(Received 9 June 2005; accepted 20 September 2005; published online 24 October 2005)

\begin{abstract}
We demonstrate the bistable lasing in a photonic molecule consisting of twin microdisks. When the photonic molecule was nonuniformly photopumped, the bistability and/or mode switching were observed with an effective pump power of $<100 \mu \mathrm{W}$. The rate equation analysis indicated that the operation was based on saturable absorption and gain switching, which depended on the nonuniformity, disk diameter detuning, and $Q$ factors of coupled modes. Such a bistable laser, particularly showing the mode switching, will be applied for ultrasmall high-speed optical memories, flip-flops, and so on. (C) 2005 American Institute of Physics. [DOI: 10.1063/1.2120906]
\end{abstract}

The microdisk laser (MDL) realizes the ultrasmall cavity, ${ }^{1,2}$ ultralow threshold, ${ }^{3,4}$ and high-speed spontaneous emission $^{4}$ by the simple geometry. But due to the strong optical confinement by the total internal reflection, this device is suitable not for extracting high output power but for applications directly utilizing localized light. In the previous study, we demonstrated photonic molecule lasers consisting of evanescent-coupled two or more MDLs. ${ }^{5}$ The photonic molecule is an optical analog to chemical molecules; modal states similar to electron energy states are discussed for coupled resonators. ${ }^{6-8}$ We observed the continuous-wave (cw) lasing in the MDL photonic molecule by photopumping, and the anticrossing characteristics and miniband formation by coupled modes. In this study, we obtained the bistable operation as an active function of the photonic molecule. Many conventional bistable semiconductor lasers utilize the saturable absorption as an operation mechanism. ${ }^{9-11}$ In coupled cavity lasers, the switching between coupled modes takes place due to the gain switching. ${ }^{12}$ These lasers have been expected to be an optical memory, flip-flop, and other functional devices. However, their large size of $100 \mu \mathrm{m}$ order and operating power of $10 \mathrm{~mW}$ order have disturbed the applications for large-scale sophisticated optical signal processing. In this letter, we demonstrate lowpower operation of $<100 \mu \mathrm{W}$ in twin MDLs of several microns in size. First, we show the fabrication process and measurement of the lasing characteristics. Next, we show the bistability and/or mode switching in both the rate equation analysis and the experiment. Finally, we discuss the critical condition that determines the bistable behaviors and response speed.

The fabrication process was the same as that in Ref. 5. A 1.55- $\mu \mathrm{m}$-GaInAsP compressively strained five-quantumwell wafer was prepared, and the device structure was formed by using $e$-beam lithography, lift-off process, $\mathrm{Cl}_{2} / \mathrm{Xe}$ inductively coupled plasma etching, and $\mathrm{HCl}$ wet etching. The scanning electron micrograph of $2.6-\mu \mathrm{m}$-diam device is shown in Fig. 1(a). The interdisk spacing was typically $0.1-0.2 \mu \mathrm{m}$. The device was photopumped at room temperature by cw laser light of $0.98 \mu \mathrm{m}$ wavelength and $20-\mu \mathrm{m}$-diam area (almost flat intensity profile due to the focused spot of light output from multimode fiber). Light

${ }^{\text {a)} E l e c t r o n i c ~ m a i l: ~ b a b a @ y n u . a c . j p ~}$ output from the device was detected by adjacent optical fiber and analyzed by optical spectrum analyzer. Figures 1(b) and 1(c) show the near-field pattern and the lasing spectrum for the even pumping of two MDLs, respectively. The effective threshold pump power $P_{\text {eff }}$ estimated by considering the effective pump area and absorption efficiency was typically 50-100 $\mu \mathrm{W}$. In the spectrum, two mode peaks are observed, which correspond to, what we call, the bonding mode on the longer-wavelength side with lower $Q$ and the antibonding mode on the shorter-wavelength side with higher $Q$. Although diameters of the two MDLs were almost the same, the field profile looks to be unevenly distributed.

In the experiment described below, we intentionally introduced an asymmetry to the device; diameters of the two MDLs were designed and fabricated to be slightly different. In actual devices, the diameter difference $\Delta 2 r$ of $24 \pm 8 \mathrm{~nm}$ was observed. For them, we performed the nonuniform pumping, in which one MDL was pumped almost entirely, while the other MDL was pumped partially. The nonuniformity was controlled by moving the focused spot of the pump light by piezostage. To avoid the thermal effect and the deterioration of the device under $\mathrm{cw}$ operation, the device temperature was lowered to $-10{ }^{\circ} \mathrm{C}$. Measured results of one sample are shown in Fig. 2. Here, the entire and partial pumpings were done for larger and smaller MDLs in (a), respectively, and vice versa in (b). Depending on the pump position, the lasing characteristic gradually changed. The standard lasing was observed in (a), while the bistable lasing with sufficiently sharp hysteresis within the resolution limit
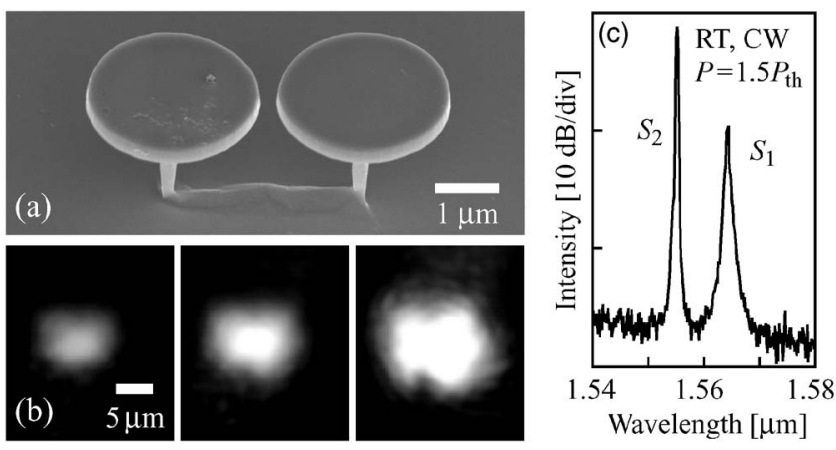

FIG. 1. Fabricated photonic molecule and its emission characteristics. (a) Top view. (b) Near-field patterns for different pump levels. (c) Lasing spectrum. 

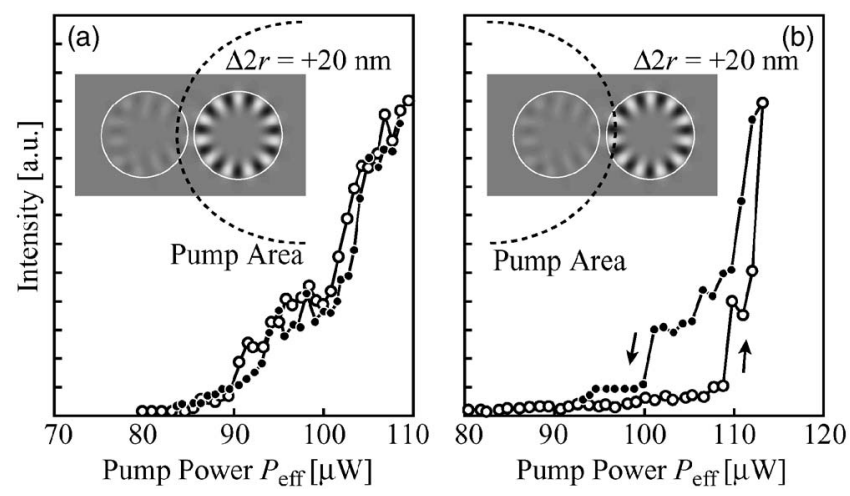

FIG. 2. Measured lasing characteristics of antibonding mode when entire and partial pumpings were carried out for (a) larger and smaller MDLs, respectively, and (b) vice versa. The open and closed circles denote modal intensities measured when increasing and decreasing the pump intensity, respectively. Lateral axis is taken by assuming that two MDLs are uniformly irradiated by pump light. However, only $10 \%-40 \%$ area of one MDL was actually pumped. Therefore, the lateral axis is $30 \%-45 \%$ overestimated than exact values. The inset shows the calculated field profiles in MDLs with different diameters.

of this measurement was observed in (b). In both cases, the lasing was obtained only for the antibonding mode having a higher theoretical $Q$. These results show that the lasing was influenced by the asymmetry of the device and the directionality of the nonuniform pumping. The insert of Fig. 2 shows the field profile of the antibonding mode calculated for $\Delta 2 r$ $=20 \mathrm{~nm}$ by the finite-difference time-domain (FDTD) method. The antibonding (bonding) mode is mainly localized in the larger (smaller) MDL. It is confirmed from the uneven distribution of the near-field pattern, as shown in Fig. 1(b). Therefore, the effective nonuniform pumping was achieved only in case (b), which resulted in the bistability.

Rate equations were used for calculating lasing characteristics. Let us denote photon densities of the bonding and antibonding modes as $S_{1}$ and $S_{2}$, respectively, and carrier densities in the MDL1 and MDL2 as $N_{1}$ and $N_{2}$, respectively. Since the resonant frequencies $\omega$ of the coupled modes are almost the same and the carrier distribution is almost uniform in each MDL due to the carrier diffusion, rate equations are approximately given by

$$
\begin{aligned}
\frac{d N_{i}}{d t}= & P_{i}-\frac{N_{i}}{\tau_{c}}-\frac{c}{n_{g}} G\left(N_{i}, N_{0}\right)\left(1-\varepsilon \sum_{j} \gamma_{i j} S_{j}\right) \sum_{j} \gamma_{i j} S_{j}, \\
\frac{d S_{j}}{d t}= & C B\left(\sum_{i} \gamma_{i j} N_{i}^{2}\right) \\
& +\frac{c}{n_{g}}\left\{\sum_{i} \gamma_{i j} G\left(N_{i}, N_{0}\right)\left(1-\varepsilon \sum_{j} \gamma_{i j} S_{j}\right)\right\} S_{j}-\frac{S_{j}}{\tau_{j}},
\end{aligned}
$$

where $i$ and $j$ equal 1 or $2, P_{i}$ is the pump rate density, $\tau_{c}$ is the carrier lifetime, $c$ is the light velocity, $n_{g}$ is the group index, $G$ is the gain coefficient as a function of $N_{i}$ and the transparent carrier density $N_{0}, \varepsilon$ is the gain saturation coefficient, $C$ is the spontaneous emission factor, $B$ is the radiative recombination coefficient, and $\tau_{j}$ is the photon lifetime given by $n_{g} Q_{j} / \omega c$ for the $Q$ factor of mode $j$. Here, $Q_{j}$ is not the ideal cavity $Q$ but that affected by the parasitic loss caused by the free-carrier absorption and the scattering loss at rough sidewalls in actual devices. The coefficient $\gamma_{i j}$ denotes the ratio how $N_{i}$ contributes to $S_{j}$ (therefore, $\gamma_{12}=1$
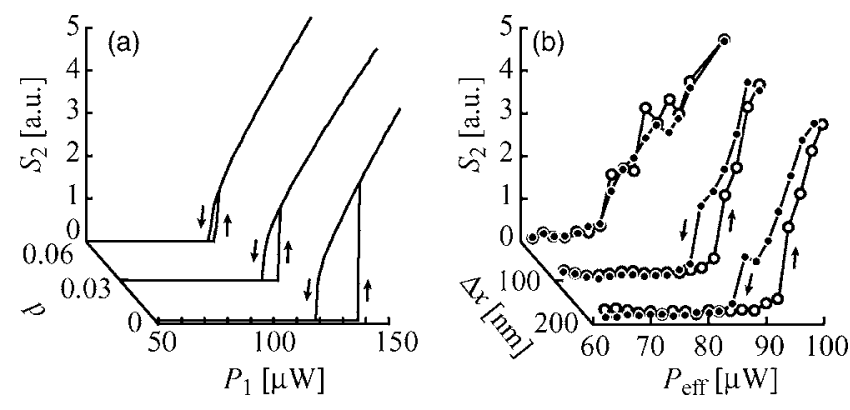

FIG. 3. Bistable lasing characteristics of antibonding mode $S_{2}$. (a) Calculated result. (b) Measured result. The open and closed circles denote the same as in Fig. 2.

$-\gamma_{11}$ and $\left.\gamma_{21}=1-\gamma_{22}\right)$. It depends on the uneven distribution of each mode described above and is 0.5 when the distribution is even. In addition, the FDTD calculation showed that $\gamma_{11}$ and $\gamma_{12}$ are almost equal to $\gamma_{22}$ and $\gamma_{21}$, respectively.

In actual calculations, we assumed $N_{0}=1.5 \times 10^{18} \mathrm{~cm}^{-3}$, $G=117 \mathrm{~cm}^{-1}$ at $N=2.5 \times 10^{18} \mathrm{~cm}^{-3}, \quad C=0.001, \quad B=3.3$ $\times 10^{-10} \mathrm{~cm}^{3} \mathrm{~s}^{-1}$, and $\varepsilon=5.0 \times 10^{-16} \mathrm{~cm}^{3}$, as typical values for GaInAsP compressively strained quantum well devices. ${ }^{4}$ In addition, we considered the situation that MDL2 was slightly larger than MDL1, and set $\gamma_{11}=\gamma_{22}=0.53$ and $\gamma_{12}=\gamma_{21}$ $=0.47$. We also set $Q_{1}=640$ and $Q_{2}=880$ in the first calculation by considering the parasitic loss to be $40 \mathrm{~cm}^{-1}$. The result for the entire pumping of MDL1 and partial pumping of MDL2 is shown in Fig. 3(a). Here, the partial pumping ratio for MDL2 is expressed by the ratio $p$, which is defined as $P_{2}=p P_{1}$. Due to the different $Q$ factors, only $S_{2}$ shows the lasing. As $p$ decreases, it exhibits the bistability more clearly with the anticlockwise hysteresis, because MDL2 acts as a saturable absorber. Figure 3(b) shows the experimental result. Similar to the calculated one, only $S_{2}$ lased and the anticlockwise hysteresis of the bistability expanded when the relative pump position $\Delta x$ was changed so that the nonuniformity increased. Such a characteristic was observed repeatedly in many samples. Next, we slightly increased $Q_{1}$ to 718 in the calculation. Then, the result changed, as shown in Fig. 4(a). Here, $S_{1}$ shows lasing with the clockwise hysteresis at the lower pump level, while the mode switching occurs due to the gain saturation and $S_{2}$ shows lasing with the counterclockwise hysteresis at the higher pump level. Figure 4(b)
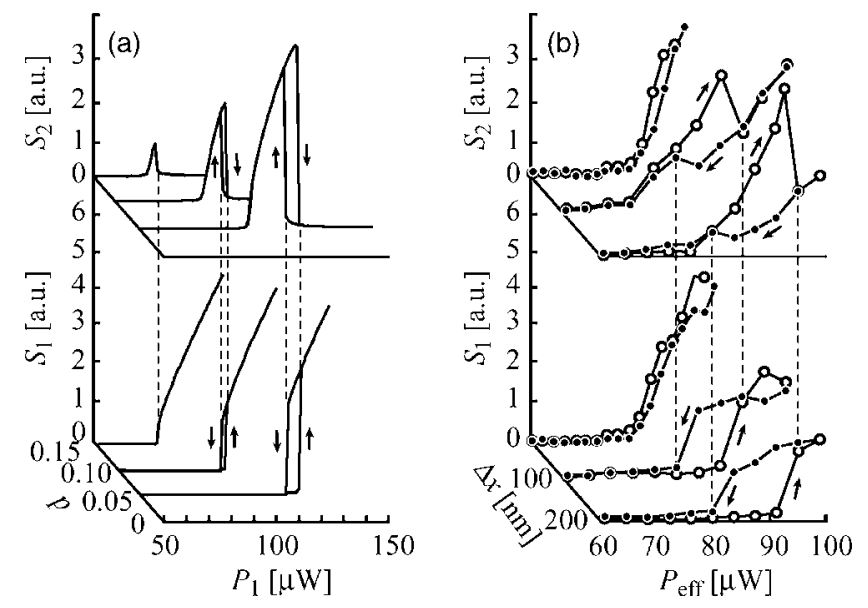

FIG. 4. Lasing characteristics showing bistable mode switching between bonding mode $S_{1}$ and antibonding mode $S_{2}$. (a) Calculated result. (b) Measured result. The open and closed circles denote the same as in Fig. 2. 


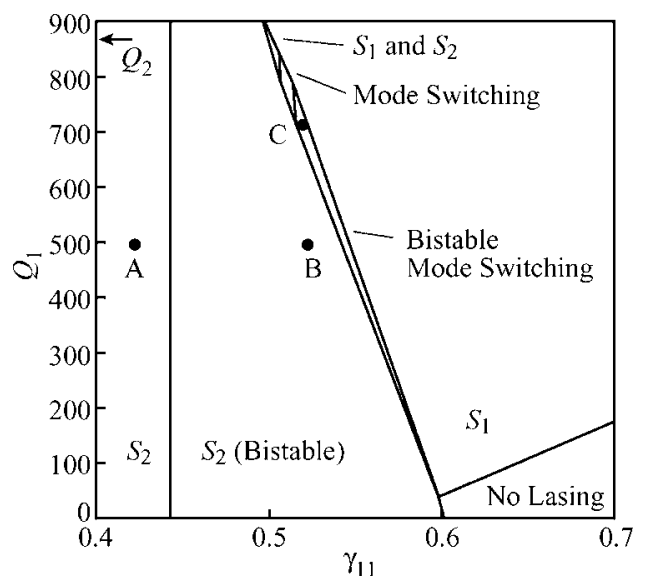

FIG. 5. Various lasing characteristics calculated for $Q_{1}$ and $\gamma_{11}$ when $Q_{2}$ $=880$ and $p=0.05$ are assumed. A, B, and C indicate the conditions which are considered to correspond Figs. 2(a), 2(b), and 4, respectively.

shows the experimental result observed in the limited number of samples, which corresponds to the calculation.

The rate equation analysis explained the condition required for the bistability and mode switching, as shown in Fig. 5. Here, we fixed $Q_{2}=880$ and $p=0.05$ and changed $Q_{1}$ and $\gamma_{11}$. For $\gamma_{11}<0.44$, only $S_{2}$ with higher $Q$ shows the standard lasing. This corresponds to the situation in Fig. 2(a). For $0.44<\gamma_{11}<0.5, S_{2}$ shows the bistable lasing due to the saturable absorption. For $\gamma_{11}>0.5$, the behavior strongly depends on $Q_{1}$. When $Q_{1}$ is too high, only $S_{1}$ shows the standard lasing. When $Q_{1}$ is too low, only $S_{2}$ shows the bistable lasing or no lasing occurs in both modes. The former corresponds to the situations in Figs. 2(b) and 3(b). The individual lasing in both modes, the simple mode switching, or the bistable mode switching, as shown in Fig. 4, are obtained when $Q_{1}$ is moderately high and the contribution of $N_{1}$ is slightly biased to $S_{1}$. In the same analysis, the dynamic response was also investigated for each condition. In the bistable lasing of $S_{2}$ based on the saturable absorption, the turn-off response is restricted by $\tau_{c}$ of several nanoseconds. In the mode switching, on the other hand, we can expect a much faster response based on the stimulated emission. Also in this case, a turn-off time of $70 \mathrm{ps}$ limits the total response because the pump intensity cannot be less than zero.

In conclusion, we observed the bistability and/or mode switching in the photonic molecule of GaInAsP twin MDLs by the nonuniform photopumping, which were well explained by the rate equation analysis. The measured threshold was less than $100 \mu \mathrm{W}$, which was extremely lower than those of bistable lasers ever reported. These characteristics are sensitive to the directionality of the nonuniform pumping, diameter of each MDL, and $Q$ factor of each coupled mode. Fundamental mechanisms are the saturable absorption or the gain switching. Particularly, high-speed operation with a response time of $70 \mathrm{ps}$ is expected for the mode switching.

This work was supported by The IT Program and The 21st COE Program of MEXT, The Grant-In-Aid of JSPS, and CREST Project of JST.

${ }^{1}$ S. L. McCall, A. F. J. Levi, R. E. Slusher, S. L. Pearton, and R. A. Logan, Appl. Phys. Lett. 60, 289 (1992).

${ }^{2}$ T. Baba, M. Fujita, A. Sakai, M. Kihara, and R. Watanabe, IEEE Photonics Technol. Lett. 9, 878 (1997).

${ }^{3}$ M. Fujita, R. Ushigome, and T. Baba, Electron. Lett. 36, 790 (2000).

${ }^{4}$ T. Baba and D. Sano, IEEE J. Sel. Top. Quantum Electron. 9, 1340 (2003).

${ }^{5}$ A. Nakagawa, S. Ishii, and T. Baba, Appl. Phys. Lett. 86, 041112 (2005).

${ }^{6}$ M. Bayer, T. Gutbrod, J. P. Reithmaier, and A. Forchel, Phys. Rev. Lett. 81, 2582 (1998).

${ }^{7}$ T. Mukaiyama, K. Takeda, H. Miyazaki, Y. Jimba, and M. KuwataGonokami, Phys. Rev. Lett. 82, 4623 (1999).

${ }^{8}$ S. Deng, W. Cai, and V. N. Astratov, Opt. Express 12, 6468 (2004).

${ }^{9}$ H. Kawaguchi, Appl. Phys. Lett. 45, 1264 (1984).

${ }^{10}$ M. Ueno and R. Lang, J. Appl. Phys. 58, 1689 (1985).

${ }^{11}$ Y. Hori, H. Sato, H. Serizawa, and T. Kajiwara, J. Appl. Phys. 60, 534 (1986).

${ }^{12}$ G. P. Agrawal and N. K. Dutta, J. Appl. Phys. 56, 664 (1985). 